\title{
Design and Implementation of a Comprehensive Real-time Java Virtual Machine
}

\author{
Joshua Auerbach \\ IBM Research \\ josh@us.ibm.com \\ Perry Cheng \\ IBM Research \\ perryche@us.ibm.com \\ David Grove \\ IBM Research \\ groved@us.ibm.com
}

\author{
David F. Bacon \\ IBM Research \\ dfb@watson.ibm.com \\ Michael Dawson \\ IBM Software Group \\ michael_dawson@ca.ibm.com \\ Darren Hart \\ IBM Linux Technology Center \\ dvhltc@us.ibm.com
}

\author{
Bob Blainey \\ IBM Software Group \\ blainey@ca.ibm.com \\ Mike Fulton \\ IBM Software Group \\ fultonm@ca.ibm.com \\ Mark Stoodley \\ IBM Software Group \\ mstoodle@ca.ibm.com
}

\begin{abstract}
The emergence of standards for programming real-time systems in Java has encouraged many developers to consider its use for systems previously only built using $\mathrm{C}$, Ada, or assembly language. However, the RTSJ standard in isolation leaves many important problems unaddressed, and suffers from some serious problems in usability and safety.

As a result, the use of Java for real-time programming has continued to be viewed as risky and adoption has been slow.

In this paper we provide a description of IBM's new real-time Java virtual machine product, which combines Metronome realtime garbage collection, ahead-of-time compilation, and a complete implementation of the RTSJ standard, running on top of a custom real-time multiprocessor Linux kernel.

We will describe the implementation of each of these components, including how they interacted both positively and negatively, and the extensions to previous work required to move it from research prototype to a system implementing the complete semantics of the Java language. The system has been adopted for hard real-time development of naval weapons systems and soft real-time telecommunications servers. We present measurements showing that the system is able to provide sub-millisecond worst-case garbage collection latencies, 50 microsecond Linux scheduling accuracy, and eliminate non-determinism due to JIT compilation.
\end{abstract}

\section{Categories and Subject Descriptors}

D.3.4 [Programming Languages]: Processors-Memory management (garbage collection); D.4.7 [Operating Systems]: Organization and Design-Real-time systems and embedded systems

\section{General Terms}

Experimentation, Languages, Performance

Permission to make digital or hard copies of all or part of this work for personal or classroom use is granted without fee provided that copies are not made or distributed for profit or commercial advantage and that copies bear this notice and the full citation on the first page. To copy otherwise, to republish, to post on servers or to redistribute to lists, requires prior specific permission and/or a fee.

EMSOFT'07, September 30-October 3, 2007, Salzburg, Austria.

Copyright 2007 ACM 978-1-59593-825-1/07/0009 ...\$5.00.

\section{Keywords}

Java, JVM, Garbage Collection, Real Time, JIT, AOT

\section{INTRODUCTION}

Real-time systems have reached a critical inflection point in both their breadth of use and the complexity of their design. The small stand-alone real-time applications of the past are giving way to a new type of networked real-time system incorporating many individual components across numerous machines, whose timeliness requirements range from microseconds to seconds, and whose aggregate size is in the tens of millions of lines of code. Examples of such systems include integrated battleship management, VOIPbased telecommunications systems, stock arbitrage, and automotive systems.

Because of the size, complexity, and longevity of these systems, there has been increasing desire (and pressure) to implement them in Java. However, until now this had not been practical.

Some pieces to the puzzle existed: our Metronome system [5] demonstrated that it was possible to build a truly real-time garbage collector, eliminating the largest obstacle to using standard Java code for real-time programming. The Real-Time Specification for Java (RTSJ) standard [9] provided much-needed real-time scheduling APIs. Unfortunately it incorporated non-standard memory management (Scopes) and was oriented towards J2ME-based uniprocessor embedded devices. The RTSJ also left key issues such as class loading and JIT compilation completely unaddressed.

In this paper we describe our design and implementation of the first production Java virtual machine to solve these issues in a comprehensive manner. The IBM Real-time Java virtual machine combines

- real-time garbage collection,

- ahead-of-time compilation,

- a complete implementation of the RTSJ standard,

- pre-loading of classes,

- customized standard libraries,

- sub-microsecond vertical instrumentation,

- full J2SE support,

- multiprocessor capability, and

- co-existence of multiple real-time JVMs on a single host.

It runs in guaranteed real-time mode on top of a real-time Linux kernel developed by IBM and the open source community, and in soft real-time mode on top of a standard Linux kernel. 
The integration for the first time of all these real-time technologies into a single system provided unique challenges and required considerable extensions and adaptations of existing techniques.

Because of the large number of components, we will of necessity be unable to treat any one of them in detail. Instead we will focus on the novel aspects of the system which were required to make all of the different components work together, and to have each piece of technology implement the complete semantics of both the complete Java 5 language as well as the complete RTSJ specification. This includes the aspects of the underlying operating system that were developed collaboratively (with the IBM Linux Technology Center) to provide the necessary features for the JVM.

The cumulative effects are shown in an experimental evaluation of the system across a number of different benchmarks.

The resulting product has been adopted by Raytheon to produce the software for DDG 1000, the next-generation U.S. Navy destroyer. It is also being used by others in the government, financial, and telecommunications sectors.

In its guaranteed real-time mode, worst-case garbage collection latencies of $2 \mathrm{~ms}$ and worst-case Linux context-switching latencies of $50 \mu \mathrm{s}$ are contractually guaranteed to customers. In this paper, we have avoided the use of the term "hard real-time" due to the potentially different interpretations of that term: in particular, whether the worst-case guarantees have been mechanically proven (as with abstract interpretation), manually proven (as with a pen-and-paper proof), certified via codified "best practices" (as with DO-178B), guaranteed contractually (as with our system), or evaluated based on test coverage. Some aspects of our system have been dealt with more formally, as we describe below.

Real-time Java is being used in environments where a new generation of real-time systems are being built, whose complexity is vastly greater than traditional real-time systems that provide localized control of single systems. Such systems have the property that their complexity level precludes the use of techniques like model checking for all but the smallest "kernels" of the system. Java is compelling for such systems since its higher level of abstraction reduces the likelihood of both bugs and security vulnerabilities.

\section{THE J9 VIRTUAL MACHINE}

The IBM Java Virtual Machine (JVM) is called J9. The core components in the JVM are the interpreter, Just-In-Time (JIT) compiler, garbage collector, class loader and stack walker. There are interfaces between all of these components. For example, when a class is loaded, the class loader updates structures used by the garbage collector, interpreter, and JIT compiler, as well as code generated by the compiler. Similarly, when the garbage collector unloads a class, the JIT is notified so that it can update it's internal structures. The code generated by the JIT is dependent on the type of garbage collector that is present.

The J9 JVM is designed to be portable, and runs on dozens of variants of operating systems and hardware combinations. The way we can achieve this high level of portability is through a very small portability layer. This portability layer provides a mapping from the general services needed by the JVM, such as acquiring memory, writing files, etc., to the underlying operating system and hardware.

The Real Time JVM is built using this same philosophy. We introduce a new garbage collector called Metronome, along with a new set of interfaces that the JIT and interpreter must implement. We extend some of the core services to support the RTSJ class libraries, in particular, priority threads, scopes and immortal memory areas, and we modify our optimization strategy to support Aheadof-Time compilation along with conservative code generation that is optimized for predictability instead of pure throughput.

\section{REAL-TIME LINUX}

IBM Real-Time Java runs in two modes: soft real-time on a stock Linux kernel and guaranteed real-time on a real-time Linux kernel. In order to provide a Linux kernel which meets the real-time requirements of the RTSJ and the Real-Time JVM, the IBM Linux Technology Center (LTC) developed a customized kernel in close association with the developers of the Real-Time Java virtual machine, and the open source Linux community. The kernel modifications are available as open source [23].

While the mainline Linux OS provided some real-time features such as the SCHED_FIFO scheduler policy and partial kernel preemption, it required changes to three key areas before it could provide the capabilities required by the real-time JVM to support the Real Time Specification for Java (RTSJ) and Metronome garbage collection. The community driven PREEMPT_RT patch provided much of the needed functionality, but was in need of some refinement and hardening. The current real-time Linux OS provided by the Linux Technology Center consists of Linux 2.6.16 and the 2.6.16-rt22 PREEMPT_RT patch, with additional bug fixes, hardware enablement patches, and RTSJ compliance features.

\subsection{Locking}

Locking mechanisms are a prime source of latencies. Latencies can arise due simply to lock contention, the need to enter kernel space to acquire frequently accessed locks, or from something more critical, like a priority inversion. The mainline kernel used spinlocks internally for several structures. The real-time kernel has replaced nearly all kernel spinlocks with mutexes (or sleeping spinlocks) which allow what would otherwise be a busy spin loop to be preempted to allow needed work to get done. Early in the development cycle, the LTC worked closely with the community to help guide a third generation fast userspace mutex implementation. This enabled the acquisition of uncontended locks to occur completely in userspace, reducing the number of times a task must enter the kernel. Lastly, priority-inheritance-enabled mutexes ensure high priority tasks block on needed locks for as little time as possible.

\subsection{Scheduling}

Even with a fully preemptable kernel, scheduling real-time tasks on an SMP system is a difficult problem. On a uniprocessor system, there is only one scheduler run queue, and selecting the next highest priority task to run is trivial. For performance reasons, the Linux scheduler maintains a separate run queue per CPU, which reduces lock contention and takes advantage of cache locality. When scheduling real-time tasks however, it is required that the $N$ CPUs on the systems be running the $N$ highest priority runnable realtime tasks at any given point in time. The LTC authored and published test cases that highlighted problems in scheduling corner cases that have since been resolved and incorporated back into the PREEMPT_RT patch. When a new real-time task becomes runnable and preempts the task running on the current CPU, the priority of the running tasks on all the remaining CPUs must be evaluated to see if the preempted task should preempt one of them. While costly, this approach ensures that strict real-time scheduling is preserved, even on an SMP system.

Interrupt handlers also contribute significantly to system latency. They typically run in kernel context, preventing anything else from using the CPU executing the handler. The real-time kernel converts the handlers to threads which can be prioritized and scheduled as a regular process. This approach provides ultimate priority control to the system designer, allowing them to specify a critical thread to run as needed even at the expense of the interrupt handlers. 
Traditionally, tasks blocked on a lock or a condition variable would be woken up and scheduled in FIFO order. In a real-time system, the tasks' relative priorities must be considered as well, and given more weight. The real-time kernel wakes tasks from these states first by priority, and then in FIFO order.

\subsection{Time and Timers}

Early 2.6 Linux kernels relied on the periodic timer tick to track time. The timer tick is an interrupt handler that would increment jiffies by one unit somewhere between 250 and 1000 times a second, depending on the configuration of the running kernel. Time was then calculated by multiplying the jiffies value by the expected duration of a jiffy and then interpolating time that had elapsed since the last tick. This method was error prone, subject to missed interrupts, variations in the system clock frequency, and abrupt NTP adjustments. Time would at times even appear to go backwards!

Real-time applications require highly accurate timekeeping in order to schedule events and track their own deadlines, which led us to rewrite the kernel timekeeping code, now also present in recent mainline kernels [32]. Most of the actual timekeeping code is moved into generic code, requiring only a small amount of architecturespecific code to define a counter, frequency, and value ranking for each clocksource, enabling the system to select the best available clocksource at boot time. This clocksource abstraction replaces the timer tick as the underlying mechanism for measuring time. Not only does this new approach avoid error-prone interpolation, but it also allows for smooth frequency-based NTP adjustments.

In parallel with the development of the new generic timekeeping subsystem, Gleixner et al [21] authored a new subsystem with support for high-resolution timers (hrtimers). This new system makes a distinction between timers (which are expected to expire) and timeouts (which typically only expire in an error condition). Timeouts don't require higher resolution and continue to use the traditional tick-granular Linux timer wheel. New nanosecond-resolution hrtimers were separated from the traditional timer system and stored in a more appropriate time-ordered red-black-tree, to be processed independently from the timer tick.

Finally, a new clock events system was added to manage the various global and per-CPU interrupt sources. Similar to the clocksource, these clock event devices are small architecture-specific drivers that are managed by architecture generic code. By combining high-resolution timekeeping with high-resolution timer structures, the clock events are used to fire interrupts as needed to expire the hrtimers. This provides lower latencies without affecting the existing periodic tick and timeout infrastructure.

\section{REAL-TIME GARBAGE COLLECTION}

Metronome [5] is a real-time garbage collector that provides highly predictable latencies down to the millisecond level. The technology is different from earlier approaches to "real-time" collection in several fundamental ways.

First, Metronome's approach to collection allows virtually all of the collection work to be done asynchronously by the collector. This is in contrast to previous approaches, which often required the application threads to perform work (like copying objects or updating pointers) on behalf of the collector, causing uneven and unpredictable performance of the application threads.

Second, Metronome takes advantage of this decoupling and uses time-based scheduling of garbage collector work, where the collector (when it is active) runs at regularly scheduled intervals of regular sizes. This makes the impact of the collector on the application highly predictable and reliable. By contrast, previous approaches typically performed work-based collection, where a unit of collector work was performed in response to application activity, for instance for every $4 \mathrm{~KB}$ of memory allocated. Since allocation is typically unevenly distributed, work-based scheduling results in uneven interruptions of the application.

Third, Metronome is engineered in such a way that its individual work quanta are extremely small, both in the average and worst case, to the point where its resolution is sufficiently high for the vast majority of real-time applications.

Metronome is able to guarantee its bound on both time and space usage [5] provided that the user can provide bounds on the maximum amount of live memory, and the maximum long-term allocation rate. Mann et al [24] describe a technique for computing worst-case allocation rates in the compiler.

Metronome was originally implemented as a research prototype in the Jikes RVM [1] virtual machine at IBM Research. In that system, we only addressed real-time garbage collection, but not the other features required for a complete system solution, that are described in the subsequent sections of this paper. It also did not implement certain Java language features, like weak/soft/phantom references. Most importantly, it was designed for uniprocessor systems with the assumption that the real-time application would have full control of the CPU.

In the process of implementing a production-quality Metronome collector, we discovered a number of issues that arise from supporting the complete language semantics and from interaction with RTSJ features. We also made some significant improvements to the original system, supporting SMPs and driving down worst-case latencies by another order of magnitude.

\subsection{Overclocked Scheduling}

Metronome's time based scheduler operates by providing a minimum mutator utilization or MMU [12]. The MMU specifies a time window and a percentage of the CPU time within that window which must be allocated to the application (a.k.a. the mutator). For instance, an MMU of $70 \%$ with a $10 \mathrm{~ms}$ time window means that for any $10 \mathrm{~ms}$ time period, the application will receive at least $70 \%$ of the CPU time (i.e., at least $7 \mathrm{~ms}$ ).

The original Metronome scheduler worked by always trying to perform a collection quantum that was exactly the size allocated to it in the MMU time window (using the example above, $3 \mathrm{~ms}$ ). This led to two problems: first of all, those quanta were large enough that they caused uneven distributions for certain kinds of operations, and second, if the collector made mistakes in its estimate of how long a piece of work would take, it would overshoot its quantum size, and violate its MMU contract. This then required compensation by shortening the collector quantum to include the jitter, leading to a slowdown of the collection process.

In the production system, we solved these problems by overclocking the collector scheduler. The collector runs at a nominal quantum size of $500 \mu \mathrm{s}$, and those quanta are spread out over the MMU window. Thus for the example above, we would nominally perform 6 separate quanta to perform the $3 \mathrm{~ms}$ of collector work. Those quanta are evenly spread, so that the application experiences less and more evenly distributed collector-induced jitter. Furthermore, the scheduler is able to do a much better job of guaranteeing the MMU target, since if a single quantum is larger than expected, it can simply either delay the next quantum slightly, or else perform a shorter quantum the next time.

\subsection{Stop-the-Worldlet SMP Scheduling}

Metronome was originally designed for a uniprocessor, since we expected it to be used primarily for small embedded systems. This turned out to be advantageous, because it forced us to solve the real- 
time scheduling problems without relying on an extra processor (which might not have been properly provisioned).

To our surprise, the early customers for the technology were building real-time systems on blade-based 4-way SMP servers. This necessitated adapting the technology significantly. However, because our fundamental real-time scheduling approach was sound, we were able to apply it in an SMP environment as well: all processors simply perform their collector quanta in a synchronized manner.

For the relatively small multiprocessors we targeted, the overhead of barrier synchronization is sufficiently small that it does not appreciably impact the amount of collector work that can be performed.

In order to scale properly we then had to adapt the collection algorithm to operate in parallel, especially for the long phases such as marking live data and sweeping free objects. While this constituted a significant amount of engineering work, we were able to employ mostly previously known techniques.

\subsection{Root Processing}

In garbage collection, roots are those pointers that form the initial set of pointers that are scanned to find live objects. These are primarily pointers in local variables on thread stacks and in global variables, but also include other types discussed below. The original Metronome prototype scanned all roots in a single collector quantum to obtain a correct initial snapshot. Otherwise, the assumptions of its snapshot-at-the-beginning approach would have been violated. In the production implementation root processing is considerably more complex because of the need to support very large numbers of threads and to implement the full Java language semantics which include several special types of pointers. As a result, processing all of the roots in a single collector quantum was not a viable option and various extensions were required to support incremental root scanning.

\subsubsection{Incremental Thread Stack Scanning}

In making the scanning of threads incremental, our goal was to deal with large numbers of threads rather than to support very deep stacks in a single thread. Proposals to deal with the latter problem exist [13], but for the expected workloads it was necessary and sufficient in practice to scan some integral number of threads in each collector quantum. To support scanning only a subset of the threads in each quantum, we employed a conservative (overinclusive) approximation of snapshot called fuzzy snapshot. It works as follows.

In the very first quantum of a garbage collection, root processing begins. In a program with a small number of threads, it may complete within that first quantum. In this case, a standard Yuasa write barrier [33], which causes all overwritten object references to be remembered, remains in effect until the end of the tracing phase of collection. Thus, the scanned threads and the mutable heap state (including class statics) are part of the snapshot.

If it is not possible to scan the roots of all threads in the first quantum, an additional write barrier is enabled. This barrier records object references that are stored rather than the references that they overwrite (which are recorded by the Yuasa barrier), and is turned off individually for each thread once its roots are scanned.

The second barrier ensures that no as-yet-unscanned root can pass into an already-scanned thread and thereafter become the sole path to a live object. It works because all objects must pass through some mutable heap location to reach another thread. We call this combined barrier the double barrier because it remembers both overwritten and newly stored pointers for unscanned threads (as well as just overwritten ones for scanned threads).
A fuzzy snapshot is a safe approximation of a snapshot even though it may omit roots that the snapshot would have included. These lost roots are precisely the ones that the unscanned threads discarded before they could either be scanned or recorded by the double barrier. But, since these roots no longer exist in the original thread and were never exposed to any other thread, they cannot be the sole means of keeping any objects live and so can be ignored.

\subsubsection{Other Roots}

The original Metronome implementation, like many other implementations of low-latency collectors, assumed that roots consist only of stack variables and static variables. The full Java language has several additional categories of roots, all of which have specific data structures and access patterns in the language, and some of which (finalizable objects, soft references, weak references) imply special semantics that the garbage collector must impose.

Each of these types of roots must be handled individually in terms of (1) how they must behave in relation to the double barrier, (2) how to incrementalize processing of the data structures that contain them, and (3) for the roots that have special semantics, how to preserve those special semantics while processing the roots incrementally. In practice, issue (2) is pure engineering and we do not go into detail about it here.

As a relatively simple example of the first issue, consider JNI global references. These may be created and destroyed through the action of JNI functions but are never overwritten in place. Despite that fact, we must model both creation and destruction as write operations: conceptually, the former replaces null with non-null and the latter does the opposite. Thus, the double barrier applies to these operations. JNI reference creation remembers the just-created root if the operation is performed by an unscanned thread while the barrier is active. JNI reference destruction remembers the justdestroyed root if it happens when the barrier is active.

Soft and weak references present much more complex problems. These "roots" are only scanned after all objects reachable from the starting root set have been marked. Java requires that if a weak reference points to an unmarked object, then the weak reference should be cleared. For soft references, the virtual machine is expected to clear a subset of the references in the event that there is memory pressure (but the remaining soft references and anything reachable from them must be marked live).

Because soft and weak references are scanned late, the Reference.get() method is intercepted by the virtual machine with what amounts to a special-purpose barrier. During a collection, up until clearable reference roots are scanned, any object that is returned by this method is also remembered as a root.

When the time comes to scan the clearable references, the soft references are scanned twice. In the first scan, a subset of the soft references are chosen for preservation. These become roots in a continuation of tracing (i.e., all objects reachable from those roots are marked). Because Java has a consistency requirement for soft reference clearing, it is not sufficient just to mark the immediate referents.

After the first scan of soft references, we commit the set of live reference types by setting a global flag indicating that marking of special reference types has completed. The Reference.get() operation has been modified to return null when this flag is set and the underlying reference has not been marked by the collector. In this manner, we avoid the need to clear all of the references atomically, and are able to incrementalize clearing. In the second scan of soft references and the sole scan of weak references, those references that point to unmarked objects are actually cleared, after which the bit may be reset. 


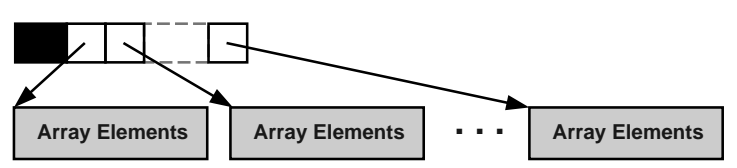

(a) Pure form: spine points to out-of-line arraylets

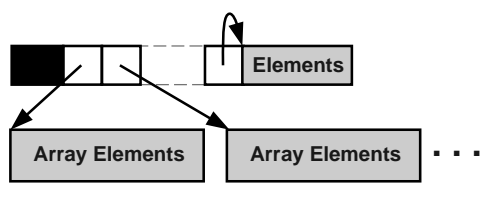

(b) Mixed form: short final arraylet is stored inside the spine

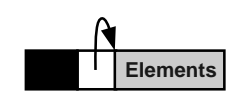

(c) Short form: all elements inside spine

\section{Figure 1: Supported variants of arraylet object model}

\subsection{Arraylets}

In order to bound the work of scanning or copying an array (one aspect of bounding collector pauses), and also to bound external fragmentation caused by large objects, Metronome relies on arraylets, a technique for breaking large arrays into chunks that can be processed in bounded time. The implementation of arraylets in a production JVM was one of the more difficult engineering tasks that we faced.

In the arraylet object model, all arrays consist of two parts: a spine, which contains the object header and pointers to arraylets, which are fixed-size chunks ${ }^{1}$ of memory that contain the array's data elements. If the last arraylet is smaller than the arraylet chunk size minus the spine header size, then it is allocated contiguously with the spine itself. This leads to three basic array organizations, as shown in Figure 1. Unlike a traditional array object model, with arraylets it is not the case that the data portion of an array is a single contiguous piece of memory.

In addition to the obvious changes in the interpreter and JIT compiler to perform the extra indexing calculations and indirection for Java-level array access, a number of other parts of the JVM and class libraries needed to be changed as well. Array manipulation, especially manipulation of byte and char arrays, is ubiquitous, and the assumption that array data was always contiguous had influenced the design of many internal APIs.

Arraylets also interacted with user and class library native code. Although the JNI functions GetArrayElements and GetPrimitiveArrayCritical include an iscopy parameter to indicate whether or not the JVM has returned a direct pointer or a copy of the argument arrays data, a common programming error is to write incorrect JNI functions that do not check this value. We discovered several such bugs in our own class libraries.

Finally, and unlike the JNI API, the two array-related functions in sun.misc.Unsafe, arrayBaseoffset and arrayIndexScale, implicitly assume contiguous arrays. To adapt this class to arraylets, we allowed the user to compute array "offsets" as if the array data was contiguous but then in the various native methods that used the offsets on array objects we first reconstruct the actual index and then do the arraylet indexing calculation. This is a suboptimal solution, but the best option available within the flaws of the established API.

\footnotetext{
${ }^{1}$ Using $2^{n}$ sized chunks allows the indexing calculation to be done with shift and mask operations. WRT currently uses $2 \mathrm{~KB}$ chunks.
}

\section{RTSJ AND REAL-TIME COLLECTION}

\subsection{NHRT null Stores}

The goal of RTSJ's No-Heap Real-Time Threads (NHRTs) is to provide a restricted programming model in which tasks can be written that can be run without interaction with or interference from the garbage collector. The NHRTs and the GC are intended to be completely decoupled. However, in the process of implementing NHRTs and Metronome, we determined that the existing specification of NHRTs has a somewhat simplistic view of potential interactions based on stop-the-world garbage collection algorithms.

NHRTs are only allowed to access immortal or scoped memory. Immortal memory is a region in which allocated objects are never collected; scopes are stacked regions whose lifetime must be manually managed by the programmer.

Although NHRTs are not allowed to read any heap references, they are allowed to overwrite memory locations (in class statics, immortal memory area, etc.) that contain heap references. This impacts the root set used for garbage collection. In the context of an incremental collector such as Metronome, the act of overwriting such a pointer incurs an obligation to notify the collector that a root has been destroyed by adding it to a write buffer. To preserve the integrity of the write buffer data structure, when the collector is removing entries from the buffer it holds a mutex. If an NHRT needs to add an entry to the buffer, it must acquire the mutex and may be delayed until the GC finishes manipulating the write buffer. This window of potential interaction can be made fairly small, and only applies to NHRTs which actually overwrite heap references, which is arguably not a common operation in well-written NHRT code. However, given that the specification allows NHRTs to overwrite heap references, we do not believe it is possible to completely avoid NHRT/collector interactions except under fairly simple GC algorithms (which are not well suited to real-time JVMs).

\subsection{GC Priority and Thread Priorities}

As is commonly done, Metronome utilizes dedicated GC worker threads to perform collection work. Our real-time Linux implementation uses priority-based scheduling. Therefore, the priorities at which the GC worker threads are allowed to run must be carefully chosen to achieve the desired scheduling of GC activities both with respect to other threads within the JVM and with respect to other JVMs and other real-time processes running on the machine.

The main constraints are: (1) GC threads must run at a strictly lower priority than NHRTs to prevent interference with an NHRT's execution, (2) GC threads must run at a priority at least as high as any heap-allocating thread (both normal Thread and Realtime Thread) in the same JVM to prevent them from being starved by the application threads whose heap allocation they are supporting and (3) it must be possible to run multiple real-time JVMs on a single processor and reason about whole-system scheduling properties.

We believe the best solution is to dynamically vary the priority of each JVM's GC worker threads such that they are marginally higher than the priority of any active heap-allocating thread within that JVM. In effect, this makes the GC threads (and thus the JVM) have the same relative priority to other processes on the system as the realtime threads within in JVM. When mapping RTSJ priorities onto Linux priorities, alternating OS priorities are reserved for Java threads and GC worker threads respectively.

Although assigning priorities in a system comprising multiple JVMs remains a complicated task, we believe that by having the JVM dynamically match its GC thread's priorities to those of its heap-allocating theads reduces the problem to "simply" assigning priorities to the Java threads. 


\subsection{Dual Barriers}

Both Metronome and RTSJ rely on read and write barriers for correct operation. These barriers represent checks and actions that must be performed before and/or after reads or writes of object fields, array elements, and global roots. In the realtime JVM, we must execute a combined barrier that performs all of the operations of the two logically distinct barriers in an appropriate order. The resulting barrier sequences are complex enough that it is infeasible to inline the entire sequence everywhere; instead just the most common short-circuit operations are done inline. A detailed discussion of the barrier sequences and some of our initial work on optimizing the common cases can be found in [19].

\section{RTSJ AND CLASS LIBRARIES}

The addition of RTSJ into a JVM adds several complications for the standard class libraries because the existing libraries were not designed with the RTSJ concepts in mind. Some areas of concern encountered during our implementation were global objects, caching, lazy initialization and consumption of immortal memory.

The class libraries provide direct or indirect access to a number of objects which are accessible to all threads within the vm. Examples include class objects, Security Managers, system properties, annotations, the default ThreadGroup and so on. Since NHRTs cannot access objects allocated from the Heap, additional work is required within the class libraries to ensure that these objects are either allocated in Immortal so that they can be directly accessed, or that there is some other way to provide the equivalent in a transparent manner. While the RTSJ identifies some of these objects (for example class objects and system properties) and mandates that they be allocated from immortal, we found that there are others that had to be identified and addressed. To identify these issues systematically we started with an analysis of static fields within our class library objects and the set of objects accessible through them.

To conserve resources, the class libraries sometimes defer initialization/creation of objects until the first time they are required. This can cause problems once RTSJ support has been added, as the current allocation context when objects are initialized/created may not be suitable. This lazy initialization can result in global objects being created from the heap such that they can later not be accessed by NHRTs, failure to store the object created if the current allocation context is a scope and the object would be stored into an object allocated from Immortal, and so on. We had to adjust a number of places within the class libraries to move the initialization/creation so that it occurred during vm initialization or so that if lazy initialization was used,the objects were created in the appropriate memory area regardless of the current allocation context.

Caching is used in the class libraries to reduce resource usage and to enhance performance. We saw several different problem with respect to caches. The first had to do with "polluted objects" in which a heap object was added to a cache by a non-NHRT and subsequently NHRTs would get a MemoryAccessError trying to use the cache. In this case NHRT access to the cache might or might not succeed depending on the objects that it needed to access from the cache. The second case was related to "polluted containers" which occurred when the expansion or reallocation of internal containers for the cache occurred in heap and all subsequent attempts by an NHRT to use the cache would fail with a MemoryAccessError. Variations of these two problems occurred when the current allocation context was a scope and IllegalStoreExceptions occurred when new objects were being added to the cache or the internal containers for the cache were being reallocated. It was necessary to either modify the operation of the caches to be safe regardless of the callers allocation context, or to disable the use of caches for NHRTs and/or threads using scopes.

\subsection{Consumption of Immortal Memory}

The Immortal Memory Area is necessary as it provides an area into which objects that need to be accessed by all threads can be allocated. However, since objects in this area are never collected it also acts as an engineered memory leak. Further, the RTSJ specifies that class objects and objects created during static initialization come from the immortal area. This ensures that Immortal is consumed even if only regular Java code is executed. It is important to consider the functionality within the class libraries such as class loading and the code executed in static initializers to minimize the use of immortal memory and, when possible, limit it to a one time hit. For example, if an object in Immortal was required so that it could be accessed by an NHRT it would often be possible to cache this object instead of creating a new copy each time.

\section{CLASS LOADING}

One of the features of Java is that it loads and initializes classes late, keeping the footprint of applications smaller and ensuring that only what is absolutely required is loaded into memory.

The notion of late binding combined with reflection and JNI makes it very challenging for Real Time Java programmers to ensure that there will be no unexpected delays due to class loading or class initialization along a particular path of code.

There are several ways to address this problem, but in practice, programmers tend to take the brute force approach of manually building a list of classes to preload, based on the classes they think are required for critical sections of their application. This is error prone and unportable, since a programmer may not have access to the source code of a particular library they want to use, and therefore may not be able to determine exactly what classes it actually depends on. Even if they do have access to the source of a particular implementation, when they move to a new version of code, or change to a new vendor, the list of classes required by a critical region could change dramatically.

An easier approach to the problem is to process all the jars, zips, and classes accessible on the command-line and pre-load all of them. This is likely to eliminate class loading from critical sections, but can take a significant amount of time and memory to process all these classes. Furthermore, there is no easy way to pre-initialize the classes that are loaded since there can be circular dependencies and hidden orderings that are required to initialize the classes correctly.

A better approach is to identify each of the critical regions of the application and apply static analysis to conservatively approximate the classes that could be referenced on any path through the region. The results of static analysis can be overly conservative because of imprecision introduced by virtual and interface method calls. The system relies on the programmer to model the affects of reflection and JNI. ${ }^{2}$ The analysis detects classes that use reflection or have native methods and require a proxy method in these cases. The proxy method mimics the methods and fields that could be reached from the original code. The static analysis code also detects which methods update static data and flag whether it has side effects. For those classes with no side effects, initialization is performed as part of class loading. The handful of classes that have side effects are not pre-initialized, but are grouped separately, in case the programmer wants to manually initialize those few classes, or, preferably, modify the code to remove the side effects.

\footnotetext{
${ }^{2}$ Programmer help is required because the only conservative assumption is that a native method could load any available class.
} 
By using the static analysis approach, either as part of deploying the application or at startup of the application, the programmer no longer needs to maintain a fragile set of classes to preload and instead can focus on the handful of proxy methods and classes with side effects. We provide this Real Time Class Analysis Tool (RaTCAT) through our alphaworks technology as a pure Java implementation that can be used with any JVM.

\section{NATIVE CODE COMPILATION}

To enable Java programs to perform at levels comparable to or better than languages like $\mathrm{C}++$ or Ada while still preserving platform neutrality, Java methods are typically compiled to native processor instructions dynamically by a Just-In-Time (JIT), compiler. Although JIT compilation is well-known to be effective in in many domains, it has two major drawbacks in the context of a real-time JVM. First, because JIT compilation occurs during program execution, compilations compete with the application for CPU resource at unpredictable times. Second, JIT compilers often employ speculative optimizations that rely on facts that are true when the compilation occurs but that might be invalidated by later program actions (thus requiring corrective actions such as patching or regenerating the compiled code). The WebSphere Real Time product includes two compilation strategies, adapted JIT compilation and AheadOf-Time(AOT) compilation, that mitigate or eliminate these nondeterministic performance effects. In the remainder of this section, we present the key ideas of these two strategies; a more detailed description can be found elsewhere [31, 19].

\subsection{JIT Compilation in a Real-time System}

The JIT compiler in the IBM WebSphere Real Time product compiles asynchronously on a separate compilation thread. To prevent it from interfering with real-time tasks, the compilation thread is given the highest non-real-time priority. The RT Linux job scheduler therefore guarantees that JIT compilations will only occur whenever there are no higher priority real-time tasks that need to be performed. Context switching times are very low in RT Linux, so this arrangement can be effective for a wide variety of real-time applications. The JIT compiler has also been modified to reduce the number of speculative optimization it performs. Only high-benefit/low-risk speculative optimizations such as speculative devirtualization and method inlining are performed. This optimization yields tremendous performance benefit, and can only be invalidated by class load events (which will be avoided during the mission-phase of a well-designed real-time application).

\subsection{Ahead-of-Time Compilation}

The RT JIT compiler should be suitable for a wide range of softer real-time applications, but there are always situations where even the RT JIT compiler will introduce too much nondeterminism to achieve the goals of a particular application. The RT AOT compiler can generate native code for methods before the program executes, which means that no JIT compiler is needed at run-time. This allows applications to achieve higher levels of deterministic performance than would be possible with simple interpretation. However, due to the dynamic nature of the Java language, code compiled ahead-of-time may not perform as well as the code generated by the RT JIT compiler. For example, class references are resolved as the program executes, so the RT AOT compiler cannot rely on the structure of a class at compile-time matching the structure of that class at run-time. A class may have a completely different implementation from one execution to the next, depending on the class path used to invoke the JVM. Alternatively, the class or a superclass may be changed between JVM invocations. For these reasons, the

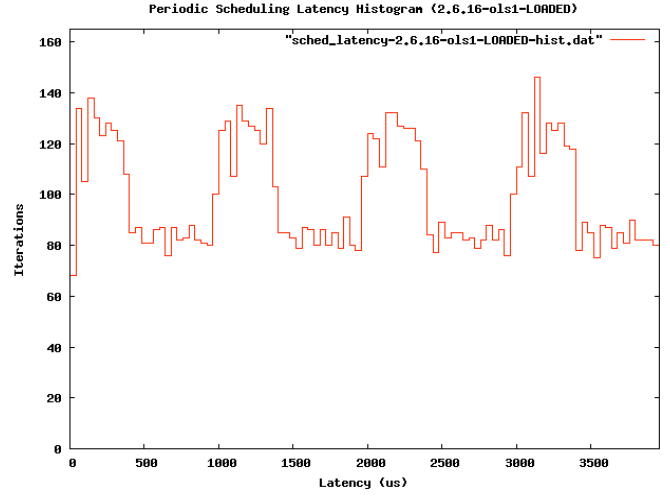

(a) Stock Linux (2.6.16)

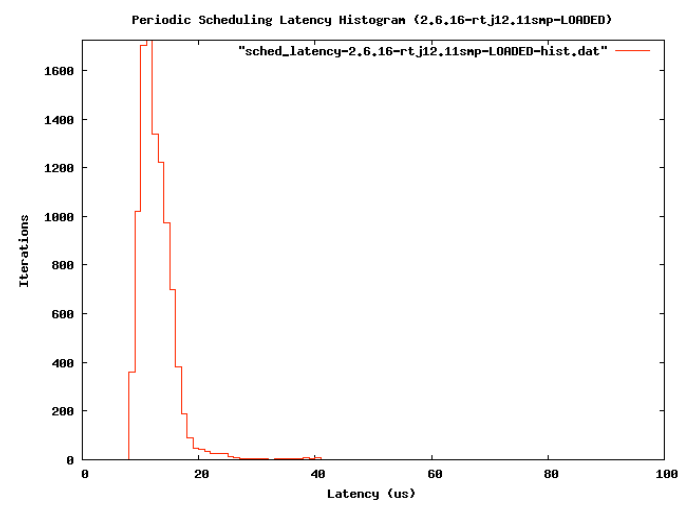

(b) IBM Real-time Linux (2.6.16 RTJ12-SMP)

Figure 2: Thread Scheduling Accuracy

RT AOT compiler cannot use information about fields, methods, or classes while generating code as effectively as the RT JIT compiler can. In particular, the AOT compiler cannot perform aggressive inlining and must generate all static, field, method, and class references as unresolved. The unresolved references can be patched by the resolution process that is tiggered the first time they execute, but the significant reduction in inlining opportunities cannot be so easily overcome.

\section{EVALUATION}

IBM Real-time Java provides contractually guaranteed real-time performance on a number of AMD-based IBM platforms. All data was collected on an IBM LS20 blade (model 8850) with two dualcore $2 \mathrm{GHz}$ AMD Opteron 270 processors, each with $1 \mathrm{MB}$ of L2 cache.

\subsection{Operating System Performance}

Figure 2 shows the effectiveness of our modifications to the Linux kernel to provide a basis for the real-time virtual machine. The histograms show the accuracy of scheduling a high-priority thread at a $5 \mathrm{~ms}$ period. 10000 iterations were run on a heavily loaded system building a new Linux kernel. The stock kernel (a) is as much as $4032 \mu \mathrm{s}$ late, with peaks at millisecond intervals corresponding to the $1 \mathrm{~ms}$ timer quantum. On the other hand, the real-time kernel (b) is always within $50 \mu$ s of the scheduled time.

\subsection{Native Code Compilation}

To evaluate the impact of native code compilation on application throughput performance, we ran the traditional raw performance 


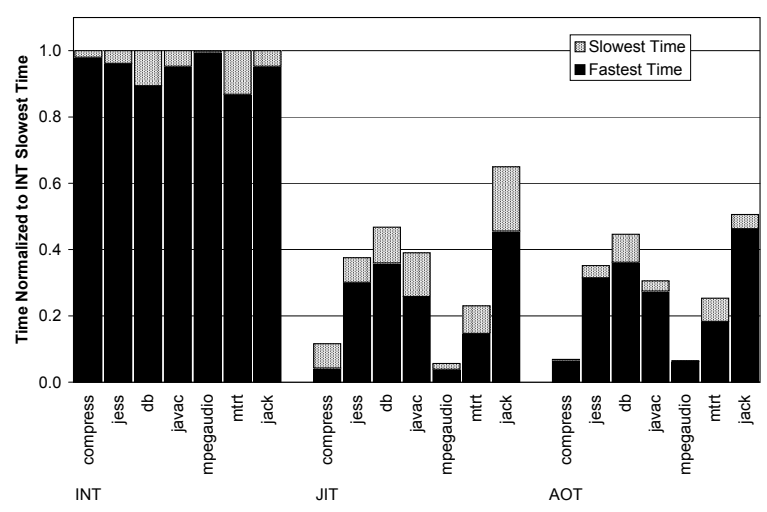

Figure 3: Interpreted (INT), Just-in-time (JIT), and ahead-oftime compiled (AOT) performance for SPECjvm98

\begin{tabular}{|l|r|r|}
\hline Benchmark & Max. Pause & MMU \\
\hline 201.compress & $650 \mu \mathrm{s}$ & $72.2 \%$ \\
202.jess & $952 \mu \mathrm{s}$ & $69.6 \%$ \\
209.db & $770 \mu \mathrm{s}$ & $69.7 \%$ \\
213.javac & $738 \mu \mathrm{s}$ & $69.4 \%$ \\
222.mpegaudio & $0 \mu \mathrm{s}$ & $100.0 \%$ \\
227.mtrt & $868 \mu \mathrm{s}$ & $70.0 \%$ \\
228.jack & $706 \mu \mathrm{s}$ & $69.3 \%$ \\
SPECjbb2000 & $920 \mu \mathrm{s}$ & $67.6 \%$ \\
\hline
\end{tabular}

Table 1: Real-time performance of garbage-collected applications. MMU is for a $10 \mathrm{~ms}$ time window

benchmarks SPECjvm98 as shown in Figure 3. There are three sets of bars for interpreter-only, JIT native code execution, and AOT native code execution. In each set, there are two overlapping bars for each benchmark. The low bar for each benchmark shows the fastest time for any execution of the test across 5 runs with 6 iterations in each run. The highest bar shows the slowest time, typically one of the first executions from one of the 5 runs. All times are normalized to the slowest interpreter run for each benchmark. As Figure 3 shows, native code compilation provides a strong performance benefit. JIT compilation provides the best improvement from $2 \mathrm{X}$ to almost $26 \mathrm{X}$ faster than the interpreter, averaging about a $9.5 \mathrm{X}$ speedup. AOT compilation provides a more modest $6.6 \mathrm{X}$ performance improvement ranging from $2 \mathrm{X}$ to almost 16X. By comparing the difference between the bars for any one benchmark, one can see that the JIT compiler's stronger throughput performance certainly incurs a penalty in determinism. Some benchmarks, such as compress and jack, improve tremendously because over time the JIT compiler can greatly accelerate the key methods. The AOT generated code, however, provides more steady performance although there are still several benchmarks we believe can be improved ( $\mathrm{db}$ and $\mathrm{mtrt}$ have the most variable performance).

\subsection{Real-time Garbage Collection}

The Metronome real-time garbage collector makes use of many of the underlying real-time Linux features, including real-time priorities, high-resolution timers, high-resolution clocks, and priority inheritance. Table 1 shows the worst-case latencies and achieved MMU for standard Java benchmarks. While these are not real-time applications, they are in fact far more allocation intensive than most

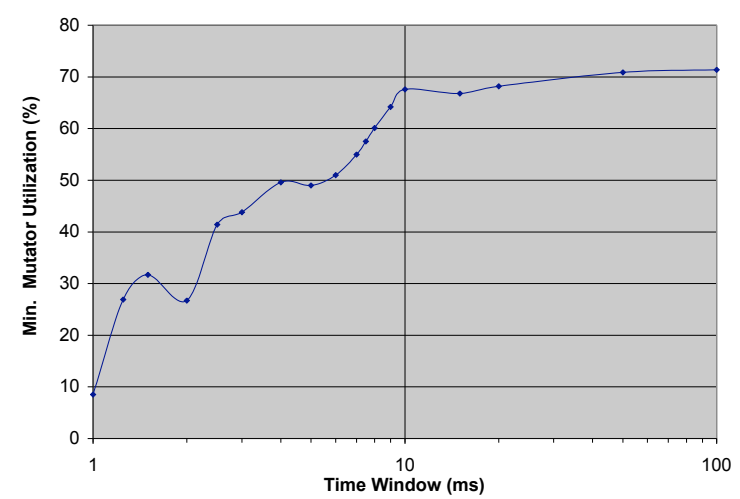

Figure 4: MMU versus time window size (SPECjbb2000)

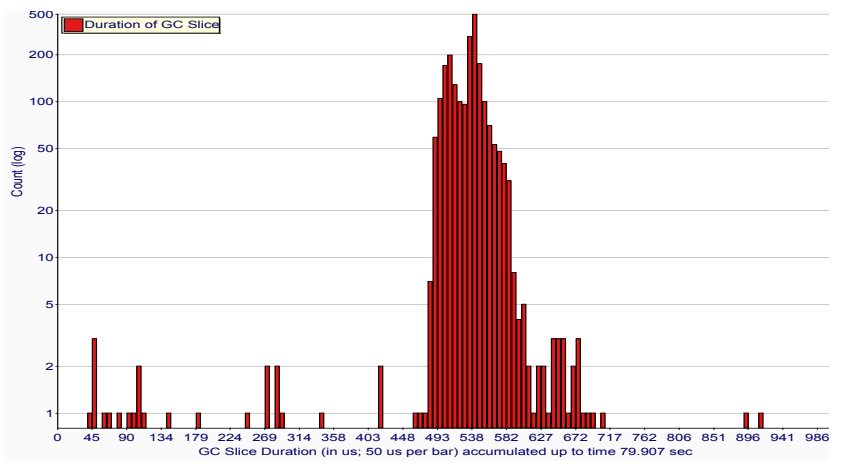

Figure 5: Collector Pause Distribution (SPECjbb2000)

such applications and thus provide a good stress test of the real-time collector. They also allow comparison with previous work [5, 14].

Worst-case pause times across all eight applications is $920 \mu \mathrm{s}$ (note that the mpegaudio benchmark triggers no collections). This is a 13-fold improvement over the original Metronome implementation in Jikes RVM [5], 23 times shorter than those reported for the hardware-assisted Azul system [14], and 163 times shorter than the times reported for BEA's "real-time" JVM [7]. Pizlo and Vitek [26] have implemented a real-time collector based on Metronome in the Ovm virtual machine with worst-case pauses of $1.8 \mathrm{~ms}$.

Minimum mutator utilization is never below $67.6 \%$ (computed over a $10 \mathrm{~ms}$ time window, with a target of $70 \%$ ). By comparison the Jikes RVM Metronome prototype only achieved 45\% MMU at $10 \mathrm{~ms}$, and the Azul collector's MMU at $10 \mathrm{~ms}$ is 0 , since it suffers $20 \mathrm{~ms}$ pauses.

Figure 4 shows the MMU behavior of SPECjbb2000 in detail, and reveals the benefits of over-clocked scheduling of the collector. A usable portion of the CPU (40\%) is already available at $2.5 \mathrm{~ms}$, reaching $50 \%$ at $5 \mathrm{~ms}$ and $67.6 \%$ at $10 \mathrm{~ms}$. By contrast, the Jikes RVM-based Metronome system could only achieve 45\% MMU at 22.2ms, while the Azul system does not execeed 50\% MMU until it reaches a $200 \mathrm{~ms}$ time window.

Figure 5 shows the distribution of pause times of collector quanta, which are tightly clustered around the nominal 500 $\mu$ s quantum, but show some jitter due to a combination of inaccuracies in the collector's work estimator, occasional longer atomic quanta, and mi- 
nor jitter in the underlying operating system scheduling (as evaluated above). However, the pauses never exceed $1 \mathrm{~ms}$, and the overclocked scheduling is able to smooth out the jitter at the quantumlevel and provide predictable MMU.

\subsection{End-to-end Transaction Time}

We now show how real-time operating system features, real-time garbage collection, thread priorities, and ahead-of-time compilation combine to produce increasingly improved real-time behavior. SPECjbb2000 transaction times are used an end-to-end metric. While such a transactional system is not representative of traditional periodic real-time systems like avionics, it is representative of many of our customer applications such as radar target tracking, financial trading, and SIP processing. We have achieved similar results for more traditional periodic real-time applications such as music synthesis and flight control.

The progression is shown in Figure 6, where the distribution of transaction times is shown up to $10 \mathrm{~ms}$, with larger outliers summarized at the right in the grey region. The y-axis is log-scaled. Figure 6(a) shows the distribution of transaction times on the standard IBM J9 JVM running on a stock 2.6.16 Linux kernel. Throughput is high with only a few outliers between 2 and $6 \mathrm{~ms}$, but there are several hundred outliers above $10 \mathrm{~ms}$. These are due mostly to garbage collection, but also to JIT compilation. With a standard JVM on top of real-time Linux (b), the distribution up to $10 \mathrm{~ms}$ is tighter, but the number of very long outliers is not much different.

Beginning with (c) we see dramatic improvements when using the real-time virtual machine. There are now only 3 outliers above $10 \mathrm{~ms}$, and 8 outliers between 2 and $10 \mathrm{~ms}$ - these are due to JIT compilation, as can be seen in (d), where the application is run with AOT compilation and worst-case transaction time drops to $1.8 \mathrm{~ms}$.

Interestingly, all of these measurements are without resorting to using real-time priorities for the application threads. The ability to do so is important because many applications in areas such as financial trading are developed in environments where reliance on the RTSJ extensions is not acceptable to users, who only wish to rely on standard Java APIs. However, we have extended our virtual machine with flags that allow the user to control the default priority for standard java.lang.Threads. Doing so further reduces the worstcase transaction time to about $1.6 \mathrm{~ms}$. Obviously, in heavily loaded environments this improvement will be more dramatic.

\section{RELATED WORK}

We have already compared our system quantitatively to existing Java virtual machines which provide some level of real-time behavior in the previous section. Azul [14] and BEA [7] are production systems that implement standard Java but not RTSJ, and provide timing 1-2 orders of magnitude worse than our system. Ovm [26] combines real-time collection and RTSJ into a single virtual machine, but is a research prototype and does not implement the complete Java language and run-time environment.

Other previous work has typically involved implementation of various components of a real-time virtual machine in isolation.

Work on real-time garbage collection began with Baker [6] and was followed by a variety of implementations with differing levels of timeliness, among them [25, 17, 3, 28, 33, 22]. Cheng [11] introduced the notion of minimum mutator utilization and built a multiprocessor collector with high observed MMU. The Metronome collector [5] upon which our system is based provided the first guaranteed MMU in concert with a strict bound on memory consumption.

A number of systems have explored static (AOT) compilation for Java. The Quicksilver project [27] persisted optimized JITed code from a training run for use in subsequent executions. Vortex [15]

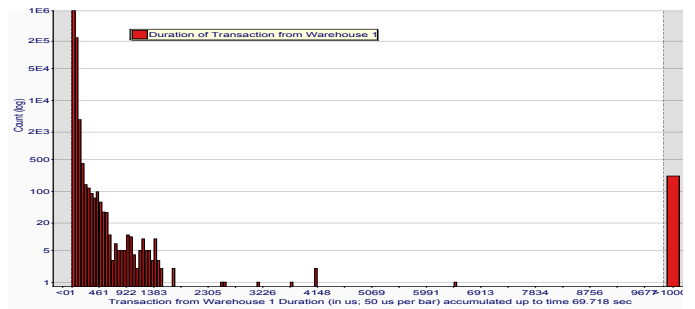

(a) Standard IBM J9 JVM on standard 2.6.16 Linux

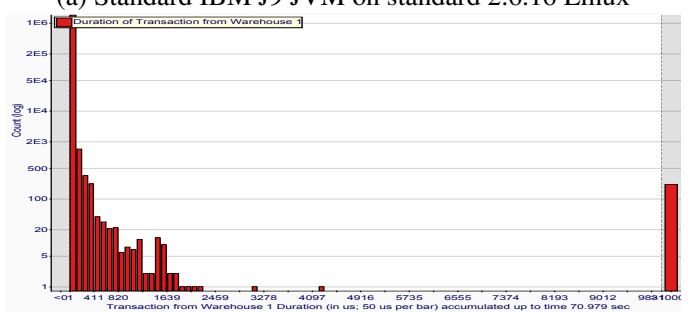

(b) Standard IBM J9 JVM on real-time Linux

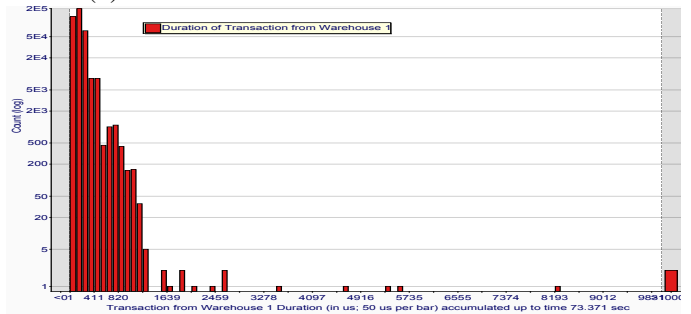

(c) Real-time JVM on real-time Linux

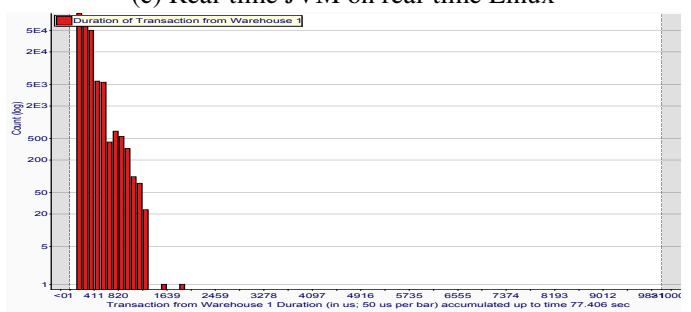

(d) Real-time JVM on real-time Linux with AOT

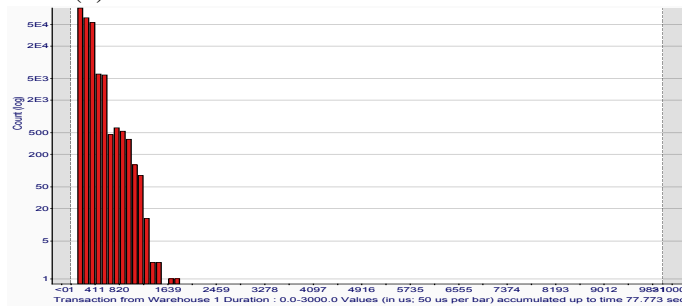

(e) Real-time JVM on real-time Linux with AOT and priorities

Figure 6: Progressive Kernel and JVM Impact on Distribution of SPECjbb2000 Transaction Times 
and Marmot [18] applied static whole program optimization Java. The GCJ project [20] has built an optimizing static compiler for Java as part of the GNU Compiler Collection. However, none of these systems were fully Java compliant.

Meanwhile work on implementing RTSJ [9] has focused on how to avoid high overheads for special checks required for its memory region checks $[4,8]$ or avoiding the cost and unreliability through various kinds of static type systems and analysis $[2,16,10]$. The difficulties of using Scopes has also led to investigation of alternative programming models capable of pre-empting the garbage collector $[29,30]$.

\section{CONCLUSIONS}

We have presented the IBM Real-time Java virtual machine, which is the first system to combine all of the features required for the construction of large-scale real-time systems in Java: real-time garbage collection, ahead-of-time compilation, and RTSJ support, and to do so in the kind of environment that the new generation of large-scale real-time systems require: full J2SE environments running on multiprocessors with multiple JVMs per host.

The resulting system achieves excellent real-time behavior and very tight bounds, which has led to its adoption for highly demanding complex real-time systems across a variety of industries.

\section{REFERENCES}

[1] AlPERn, B., ET AL. The Jalapeño virtual machine. IBM Syst. J. 39, 1 (Feb. 2000), 211-238.

[2] Andreae, C., Coady, Y., Gibbs, C., Noble, J., Vitek, J., And ZHAO, T. Scoped Types and Aspects for Real-Time Java. In Proc. European Conference on Object-Oriented Programming (Nantes, France, July 2006), Springer-Verlag, pp. 124-147.

[3] ApPel, A. W., Ellis, J. R., AND LI, K. Real-time concurrent collection on stock multiprocessors. In Proc. Conference on Programming Language Design and Implementation (Atlanta, Georgia, June 1988). SIGPLAN Notices, 23, 7 (July), 11-20.

[4] Armbuster, A., ET AL. A Real-time Java virtual machine with applications in avionics. ACM Transactions in Embedded Computing Systems (2006).

[5] Bacon, D. F., Cheng, P., And Rajan, V. T. A real-time garbage collector with low overhead and consistent utilization. In Proceedings of the 30th Annual ACM SIGPLAN-SIGACT Symposium on Principles of Programming Languages (New Orleans, Louisiana, Jan. 2003). SIGPLAN Notices, 38, 1, 285-298.

[6] BAKER, H. G. List processing in real-time on a serial computer. Commun. ACM 21, 4 (Apr. 1978), 280-294.

[7] BEA. BEA WebLogic real time: Predictable mission-critical performance for java - today. Technical white paper available at http://www.bea.com, Jan. 2006.

[8] BeEbee, W. S., AND Rinard, M. C. An implementation of scoped memory for real-time Java. In EMSOFT '01: Proceedings of the First International Workshop on Embedded Software (2001), pp. 289-305.

[9] Bollella, G., Gosling, J., Brosgol, B., Dibble, P., Furr, S., Hardin, D., AND Turnbull, M. The Real-Time Specification for Java. The Java Series. Addison-Wesley, 2000.

[10] Boyapati, C., Salcianu, A., William Beebee, J., And RINARD, M. Ownership types for safe region-based memory management in real-time java. In Proc. Conf. on Programming Language Design and Implementation (2003), pp. 324-337.

[11] Cheng, P. Scalable Real-Time Parallel Garbage Collection for Symmetric Multiprocessors. PhD thesis, Carnegie-Mellon Univ., Sept. 2001.

[12] Cheng, P., AND Blelloch, G. A parallel, real-time garbage collector. In Proc. SIGPLAN Conference on Programming Language Design and Implementation (Snowbird, Utah, June 2001). SIGPLAN Notices, 36, 5 (May), 125-136.
[13] Cheng, P., Harper, R., And Lee, P. Generational stack collection and profile-driven pretenuring. In Proc. Conference on Programming Language Design and Implementation (June 1998). SIGPLAN Notices, 33, 6, 162-173.

[14] Click, C., Tene, G., AND Wolf, M. The pauseless gc algorithm. In VEE '05: Proceedings of the 1st ACM/USENIX international conference on Virtual execution environments (2005), pp. 46-56.

[15] Dean, J., DeFouw, G., Grove, D., Livinov, V., AND CHAMBERS, C. Vortex: an optimizing compiler for object-oriented languages. ACM SIGPLAN Notices 31, 10 (Oct. 1996), 83-100.

[16] Deters, M., And Cytron, R. K. Automated discovery of scoped memory regions for real-time Java. In Proc. Third International Symposium on Memory Management (Berlin, Germany, June 2002). SIGPLAN Notices, 38, 2 Supplement, 25-35.

[17] Doligez, D., And Gonthier, G. Portable, unobtrusive garbage collection for multiprocessor systems. In Conf. Record of the Twenty-First ACM Symposium on Principles of Programming Languages (Jan. 1994), pp. 70-83.

[18] Fitzgerald, R., Knoblock, T. B., Ruf, E., Steensgaard, B., AND TARDITI, D. Marmot: An optimizing compiler for Java. Tech. Rep. MSR-TR-99-33, Microsoft Research, June 1999.

[19] Fulton, M., AND Stoodley, M. Compilation techniques for real-time Java programs. In Proc. International Symposium on Code Generation and Optimization (2007).

[20] The GNU Compiler for the Java Programming Language. http://gcc.gnu.org/java.

[21] Gleixner, T., AND Niehaus, D. HRTimers and beyond: Transforming the Linux time subsystems. In Proc. Linux Symposium (Ottawa, Ontario, June 2006).

[22] HenriksSON, R. Scheduling Garbage Collection in Embedded Systems. PhD thesis, Lund Institute of Technology, July 1998.

[23] IBM Linux Technology Center. Real-time linux patches. $\mathrm{ftp} / / /$ linuxpatch.ncsa.uiuc.edu/rt-linux/rhel4u2/R1/rtlinux-src-200608-30-r541.tar.bz2.

[24] Mann, T., Deters, M., LeGrand, R., And Cytron, R. K. Static determination of allocation rates to support real-time garbage collection. In Proc. ACM Conference on Languages, Compilers, and Tools for Embedded Systems (2005), pp. 193-202.

[25] Nettles, S., AND O'Toole, J. Real-time garbage collection. In Proc. SIGPLAN Conference on Programming Language Design and Implementation (June 1993). SIGPLAN Notices, 28, 6, 217-226.

[26] Pizlo, F., And Vitek, J. An emprical evaluation of memory management alternatives for real-time Java. In Proc. 27th IEEE International Real-Time Systems Symposium (2006), pp. 35-46.

[27] Serrano, M., Bordawekar, R., MidkifF, S., and Gupta, M. Quicksilver: a quasi-static compiler for Java. ACM SIGPLAN Notices 35, 10 (Oct. 2000), 66-82. In Conference on Object-Oriented Programming, Systems, Languages and Applications (OOPSLA).

[28] SIEBERT, F. Eliminating external fragmentation in a non-moving garbage collector for Java. In International Conference on Compilers, Architecture, and Synthesis for Embedded Systems (San Jose, California, Nov. 2000), pp. 9-17.

[29] Spoonhower, D., Auerbach, J., Bacon, D. F., Cheng, P., AND GROVE, D. Eventrons: a safe programming construct for high-frequency hard real-time applications. In Proceedings of the ACM SIGPLAN conference on Programming Language Design and Implementation (Ottawa, Ontario, Canada, 2006), pp. 283-294.

[30] Spring, J. H., Pizlo, F., Guerraoui, R., and Vitek, J. Programming abstractions for highly responsive systems. In Third International ACM SIGPLAN/SIGOPS Conference on Virtual Execution Environments (San Diego, California, 2007).

[31] Stoodley, M., Ma, K., AND LUt, M. Real-time Java, part 2: Comparing compilation techniques (2007). http://www.ibm.com/developerworks/java/library/j-rtj2/index.html.

[32] Stultz, J., Hart, D., AND Aravamudan, N. We are not getting any younger: A new approach to time and timers. In Proc. Linux Symposium (Ottawa, Ontario, June 2005).

[33] YuASA, T. Real-time garbage collection on general-purpose machines. J. Systems and Software 11, 3 (Mar. 1990), 181-198. 